\title{
Internet of robotic things: Design and develop the quality of service framework for the healthcare sector using CoAP
}

\author{
Arun Kumar, Sharad Sharma \\ Department of Electronics and Communication Engineering, Maharishi Markandeshwar (Deemed to be University), \\ Mullana, India
}

\section{Article Info \\ Article history: \\ Received May 9, 2020 \\ Revised Dec 10, 2020 \\ Accepted Jul 23, 2021}

\section{Keywords:}

Cloud computing

CoAP protocol

Contiki Cooja

IoT

Robot

\begin{abstract}
The number of robotics used globally is gradually growing, according to a variety of research. They are becoming more and more popular in different workplaces, like manufacturing, distribution, medical conditions, military, inaccessible areas, etc. The internet of things (IoT) and robotics groups have until now been guided by a set of, but somewhat compatible, goals, which are mainly to help knowledge systems in the field of general sensing, tracking, and monitoring. Therefore, the development of an internet of robotic things (IoRT), which incorporates the outcome from both cultures, is progressively said to have a significant added benefit. Internet of robotic things, the intersection of the Internet of Things and robotics, is where selfsufficient machines will assemble information from various sensors and speak with one another to perform errands including basic reasoning. As the name suggests, IoRT is the combination of two front-line innovations, the internet of things and robotics. People can manage any electronic device in homes with IoT and can also be used in contactless applications in healthcare. The constrained application protocol (CoAP), for the management and control of a community of homogeneous sensor modules, has recently endorsed multicast communications in IoRT. It will boost connectivity performance, less power consumption due to data aggregation, and enhanced security features with DTLS security features for various applications for the internet of things. This paper presents an implementation of the CoAP framework on IoRT sky motes using the Contiki Cooja Simulator that will be a useful healthcare sector that will confirm their potential and therefore, new research directions are outlined.
\end{abstract}

This is an open access article under the CC BY-SA license.

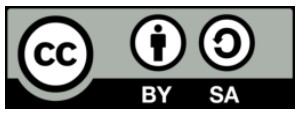

\section{Corresponding Author:}

Arun Kumar

Department of Electronics and Communication Engineering

Maharishi Markandeshwar (Deemed to be University)

Mullana, India

Email: ranaarun1@gmail.com

\section{INTRODUCTION}

The robot can be coordinated as an element in the internet of things (IoT) foundation along these lines empowering associations between various elements utilizing assorted communication protocols. Internet of robotic things (IoRT) is 'robot(s) associated as a thing' in IoT innovation which builds up associations with different things over the Internet. IoRT is brilliant robots that are incorporated through the internet for performing proficient procedures or individual exercises, for example, the assembling procedure and observing of older exercises. They are wise gadgets that help with checking occasions and controlling items in the physical world by social affair sensor information from an assortment of sources to decide the 
gameplan [1]. Developing the web-based business industry and expanding application territories inferable from the coordination of robots with different advancements is boosting the internet of automated things utilization.

The significant driving variables for the development of the worldwide internet of apply autonomy showcase are fast information networks and consistent web availability [2]-[4]. Increment in the utilization of shrewd gadgets, online business, mechanization in the assembling procedure, digitalization, old populace, ascend inaccessibility of broadband framework, and urbanization is additionally adding to the development of the worldwide Internet of mechanical autonomy things showcase. In any case, the absence of consciousness of the Internet of apply autonomy innovation, the significant expense of innovative work exercises, and unpredictability in new item improvement are the components obstructing the development of the worldwide Internet of the mechanical technology advertise. Developing necessities for access and authority over the Internet of apply autonomy innovation gadgets across businesses and the reception of modern robots by little and medium endeavors is relied upon to make open doors for merchants working on the Internet of automated things showcase. This would take into consideration further increment in clients' experience, simple coordination, and bolster administrations, for example, help and the executives of system and administrations in modern procedures.

Therefore, in combining this community, we attempt to create a new internet of robotic stuff, as illustrated in Figure 1. IoRT is an IoT, cloud, and robotics blend. IoRT acts as a global network which makes it possible for advanced robots to be linked to interoperable information from both emerging and established communication technology. Cloud robotics is a cloud-based robot that uses a shared pool of cloud resources. Cloud robotics implement unified features, delegate resources to support several robots to store and process information. One important aspect that is lacking in cloud robotics, however, is the capacity of the robot to interact with other robots and computers over the web utilizing a growing networking system. IoT is one approach that allows a robust connectivity network.

The rest of the paper is structured as follows: section 2 on the "literature survey" offers state-of-theart research on key issues in the field of IoRT. Proposed IoRT models are explored in section 3 named "research method results and discussion" which provides an analysis of results with the algorithm in section 4. The paper concluded in section 5 named "conclusion."

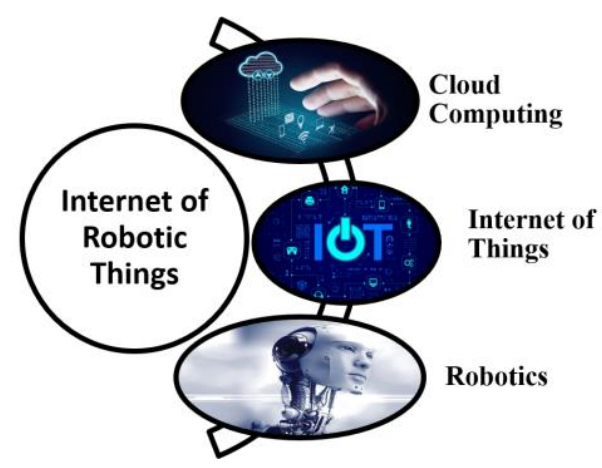

Figure 1. Internet of robotic things (IoRT)

\section{LITERATURE SURVEY}

The internet of things brings gives a digital heartbeat to physical objects and this section will discuss the historical prospect of IoRT. Robotics is a branch of computer science and engineering that deals with machines that can work autonomously. IoRT is a concept where IoT data helps machines interact with each other and take required actions. In simpler words, it refers to robots that communicate with other robots and take appropriate decisions on their own. Early indications of the IoT-robot autonomy intermingling can be seen in dispersed, heterogeneous robot control standards like system robot frameworks or robot ecologies, 5 or approaches, for example, omnipresent apply autonomy [5]-[8] and cloud robotics [9]-[12] that place asset serious highlights on the server-side.[13], [14]. The term 'Web of automated things' itself was begotten in a report of allied business intelligence (ABI) research to mean an idea where sensor information from an assortment of sources is combined, prepared to utilize nearby and disseminate knowledge, and used to control and control protests in the physical world. In this digital-physical point of view of the IoRT, sensor, and information examination advances from the IoT are utilized to give robots a more extensive situational mindfulness that prompts better undertaking execution. Use cases incorporate clever transportation [15] and 
buddy robots [16], IoT: A dream, engineering components, and future headings" addresses that universal detecting empowered by wireless sensor network (WSN) advances are introduced in numerous regions of current life [17]. Associating with the internet of things to the clouds give progressively hearty security. The usage that appeared in the article is proof of the idea of this methodology [18]. Enrolls advances behind IoRT, uses of IoRT, and existing mechanical frameworks dependent on humanoid, mobile, flying, and swarm visualized for future IoRT frameworks [19]. In numerous situations, machines and robots are assuming a significant job in the computerization business and the procedure through which the mechanical arm is made with the assistance of Arduino and a potentiometer for controlling and planning the modern procedures [20]. The framework abuses exact robot restriction data got by coordinating odometry, inertial, and global position system (GPS) estimations. IoT hubs actualize an IPv6 convention, with IEEE 802.15.4 (6TiSCH) innovation and utilize multi-bounce correspondences, to ensure an IoT organize topology that gives wide inclusion [21]. Automation systems have significant problems mainly in the health sector and their reliability concerns. Some approaches currently existing to seek to identify potential new ideas are discussed. With physical or mobile devices cyber protection threats are still present, robots are often influenced by cybersecurity threats, hackers can exploit contact between robots and surgeons [22]-[27]. This paper proposed a secure and energyefficient constrained application protocol (CoAP) framework on IoRT sky motes using the Contiki Cooja simulator that will explore future gateway for all researchers.

\section{RESEARCH METHOD}

Contiki Cooja simulator is a very good network simulator that is very useful for IoRT environments. The main feature of this simulator is based on the event-driven kernel and also supports TCP IP network with the use of uIP library. This is also useful for or protothreads programming abstraction. Contiki is implemented in the $\mathrm{C}$ language and has been designed to be easily portable to new platforms. It has been ported to more than 20 different platforms since its release in 2003. Contiki is a broadly utilized IoT empowered working framework that is free and open source. Cooja is the Contiki organize test system and is additionally used to program sensor gadgets. Peruse on to figure out how IPv6 conventions can be customized with Contiki and Cooja Security and honesty are the fundamental issues in an IoT-based system condition where capture free made sure about correspondence is required; CoAP is a very useful protocol in the present scenario of IoT. CoAP sets out four protection modes: a) NoSec, with Datagram TLS (DTLS), deactivated, b) PreSharedKey, with activated DTLS, c) raw public key, with activated DTLS, d) elliptic curve cryptography (ECC), confirmable $(\mathrm{CON})$ is the durability of signing a letter. A CON message is retransmitted by law and exponential back-off between broadcasts, to the receiver sends the message acknowledgment (ACK) with the same message ID (in that example is $0 \mathrm{x} 7 \mathrm{~d} 34$ ) from the appropriate endpoint; see Figure 2.

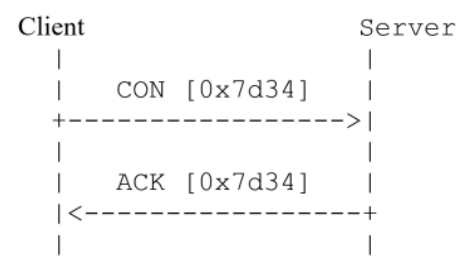

Figure 2. Reliable message transmission

If a receiver cannot process a confirmable signal at all (I.e. not even able to provide an acceptable answer to an error), replies with a reset message (RST) instead of a greeting. Many methods in IoT networks safe contact, but each one has inconvenienced. The proposed approach uses elliptic curve cryptography (ECC) methods which depend on the algebraic structure of the elliptical curves over finite fields to overcome these limitations. Internal and public keys are the primary application of elliptic curves with this method. They are also used when the messages are encrypted and decrypted. For this alternative approach, this method was used to decrease computing power since the key size of the method is the least many cryptographic algorithms in contrast.

A random number $\mathrm{R}$ in a range (n) is then chosen. Equation (1) is then used to produce the public key:

$$
P U=R * P
$$


If $\mathrm{R}$ is a random number between 1 and $\mathrm{n}-1$, and $\mathrm{P}$, the point in the curve produced by the next equation is referred to (2) and PU refers to the public key, and $n$ to the private key.

$$
y^{2}=x^{3}+a x+b
$$

This is a very light protocol for the constrained environment and is also useful to check the status of different IoRT sensors deployed in Contiki Cooja. CoAP supports server-client interface read and controls different types of the deployed sensor as shown in Figure 3.

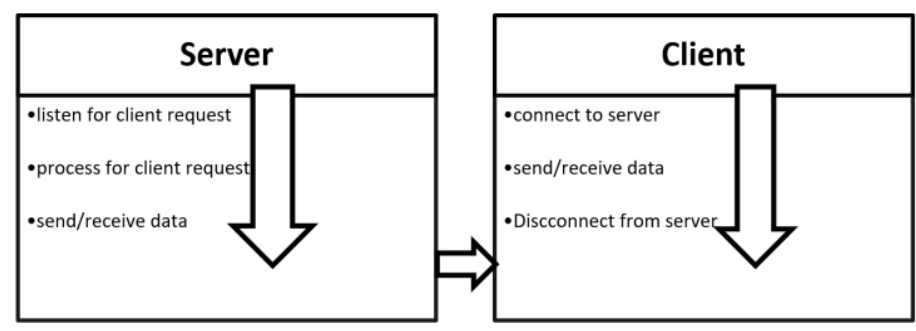

Figure 3. CoAP server and client interface

Our proposed model is shown in Figure 4 having different 20 IoRT sensor (client) that receive the information and forward to the border router (server). Then border router forwards this information to the internet having a connection IPv4 and IPv6 and with the help of any digital device like mobile (M) or PC, anyone can read the value os sensor.

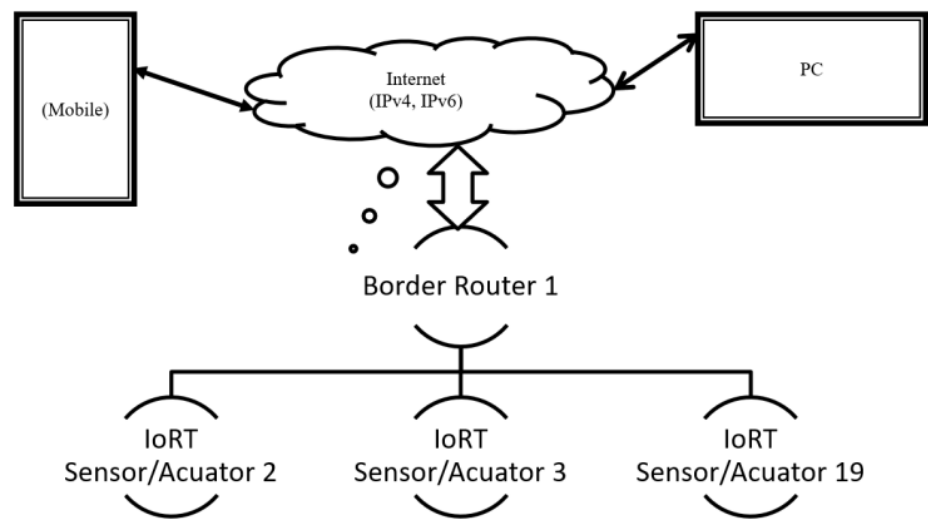

Figure 4. Proposed models of IoRT

\section{RESULTS AND DISCUSSION}

In Figure 5 arrangement of our Contiki Cooja simulation model with the sky, motes are shown and features of sky motes are discussed in the last of this section. Contiki Cooja is a very good open-source, highly portable, and multi-tasks operating system in these nodes, node 1 one act as a border router, and remaining notes, will work as executor for user datagram protocol (UDP) server code and behave as IoRT sensors. The algorithm for the simulations is shown in Figure 5. In our scenario, we can organize all nodes in different topologies with the help of drag and drops in the Contiki Cooja simulator. By clicking any node you can see a radio environment with two colors green and grey color as shown in Figure 6. In this grey color present, the scenario where radio interference maybe occurs between neighboring notes and the green environment represents the faithful environment for the reception of signal without any interface as shown in our scenario. 


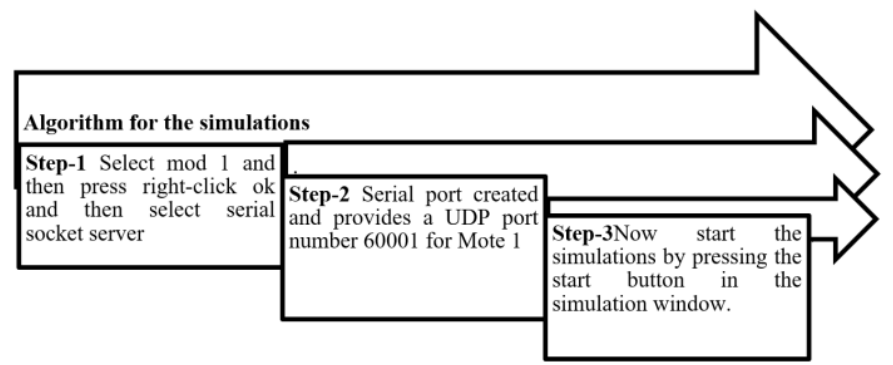

Figure 5. Algorithm for the simulations

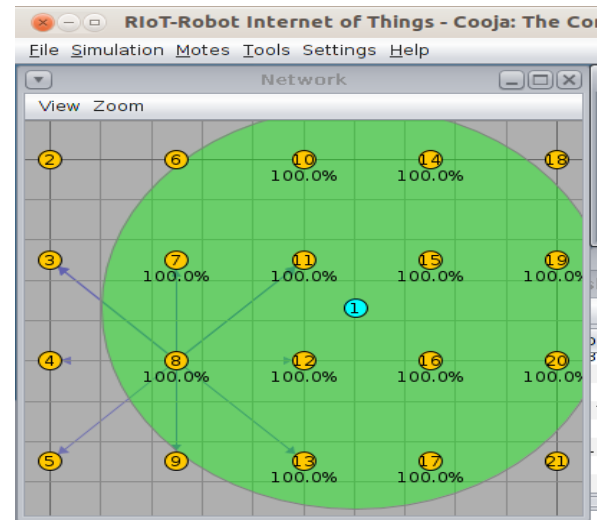

Figure 6. Screenshot after simulation in Cooja

When simulation started then copy IPv6 address [aaa::212:7401:1:101] from the command window of the border router, now paste this address in the Mozilla browser, when the IPv6 address is executed by the Mozilla browser then this will give the information of neighboring 12 notes of the bridge router that are in the green range as shown in Figure 7 and route mote also shown in the Mozilla browser which is not in the range of border router.

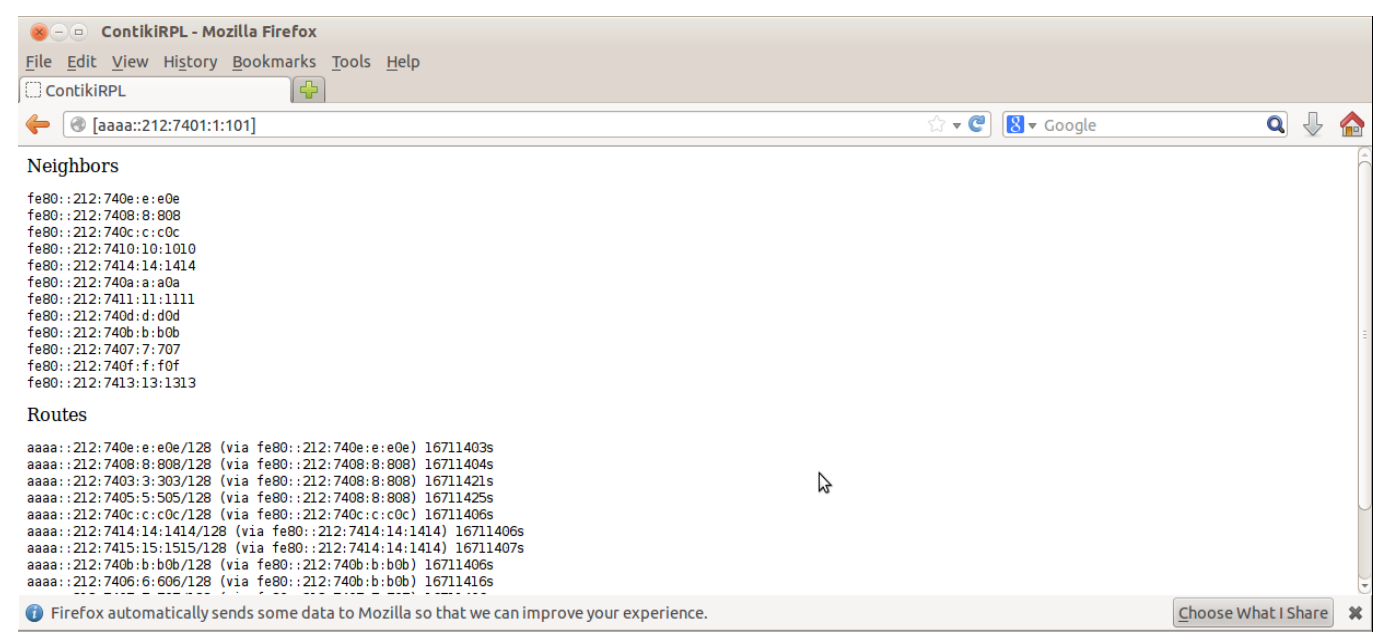

Figure 7. Route statuses, as visible to the border router

This CoAP can be gotten to comparable like hypertext transfer protocol (HTTP), to get to a specific bit (sensor) with IPv6 like this CoAP://[aaaa::212:740c:c:c0c] The above line will bring the sensor data 
peripherals and one can control from the program itself as appeared in Figure 8. It is seen that this browser provides value oo IoRT sensor as temperature 24 and light 108. Mozilla browser has a $\mathrm{Cu}$ module to empower CoAP inside the program. CoAP is now accessible for all the gadgets like iOS, android, windows, Linux, Mac, and so forth. In this way bringing about the show, constrained application protocol (CoAP) is a developing application layer convention supporting asset compelled gadgets and simple reconciliation with the current TCP/IP standard and helpful for cutting edge applications in the human services field.

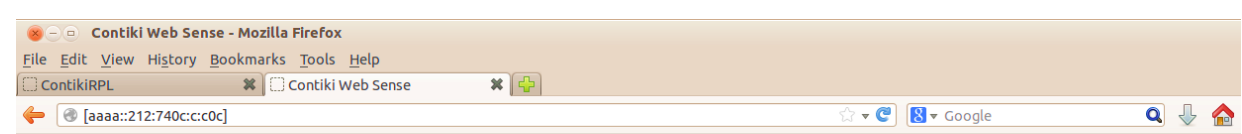

\section{Current readings}

Light: 108

Temperature: $24^{\circ} \mathrm{C}$

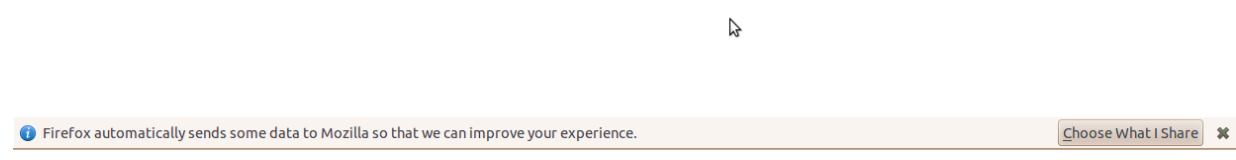

Figure 8. IoRT sensor output

The CoAP protocol is to be in the IoT network application layer and provides security services for trust, authorization, and data integrity for transferring data across the IoT network between service agents. Sky mote is a very good sensor-supported IPv6 protocol, in the field of the internet of things that provides very high data rates for very low power devices and some of the features are Support interoperability, very low current consumption in sky mote. Universal serial bus (USB) data interface, sleep and fast wake up mode facility, very useful in light humidity and temperature sensor and best networking support

\section{CONCLUSION}

Robotics and IoT are two words, covering a variety of ideas and technologies. The IoT benefits exploited by robotics are mostly distributed perception and M2 M protocols. Conversely, the IoT has used robots mainly for active sensing so far tactics. The fundamental point of this paper is the execution of the CoAP convention in the Mozilla browser program and the control of assets by CoAP strategies. The utilization of the CoAP convention for IoRT applications beats some notable issues identified with asset compelled conditions. CoAP is a solid and viable application convention, expected for use in IoT. The main motive of using a protocol is to ensure security because CoAP protocol is based on UDP to transport information that provides security ensuring the information. In this paper, we use only 21 sky mote and read the status of all nodes with help of the Mozilla Firefox browser and in the future limit of the sky mote IoRT sensor can be extended up to 1000 with the help of the Contiki Cooja simulator. This research paper will ideally inspire researchers in both disciplines to begin work on an ecosystem that incorporates the above two readings holistically, including IoT agents, robots, and the cloud.

\section{REFERENCES}

[1] A. K. Rana and S. Sharma, "Enhanced Energy-efficient heterogeneous Routing Protocols in WSNs for IoT Application," International Journal of Engineering and Advanced Technology (IJEAT), vol. 9, no. 1, pp. 44184425, 2019, doi: 10.35940/ijeat.A1342.109119.

[2] K. Kumar, S. Gupta and A. K. Rana, "Wireless Sensor Networks: A review on "Challenges and Opportunities for the Future world-LTE"," Amity Journal of Computational Sciences (AJCS), vol. 1, no. 2, pp. 30-34, 2017.

[3] A. K. Rana, R. Krishna, S. Dhwan, S. Sharma and R. Gupta, "Review on Artificial Intelligence with Internet of Things - Problems, Challenges and Opportunities," 2019 2nd International Conference on Power Energy, Environment and Intelligent Control (PEEIC), 2019, pp. 383-387, doi: 10.1109/PEEIC47157.2019.8976588. 
[4] A. Kumar, A. O. Salau, S. Gupta, K. Paliwal, "Recent Trends in IoT, its Requisition with IoT Built Engineering: A Review," in Advances in Signal Processing and Communication, vol. 526, pp. 15-25, 2019, doi: 10.1007/978-98113-2553-3_2.

[5] A. Saffiotti et al., "The PEIS-Ecology project: Vision and results," 2008 IEEE/RSJ International Conference on Intelligent Robots and Systems, 2008, pp. 2329-2335, doi: 10.1109/IROS.2008.4650962.

[6] J. Kim, K. Lee, Y. Kim, N. S. Kuppuswamy and J. Jo, "Ubiquitous Robot: A New Paradigm for Integrated Services," Proceedings 2007 IEEE International Conference on Robotics and Automation, 2007, pp. 2853-2858, doi: 10.1109/ROBOT.2007.363904.

[7] J.-H. Kim, "Ubiquitous robot," in Computational Intelligence, Theory and Applications, vol. 33, pp. 451-459, 2008, doi: 10.1007/3-540-31182-3_41.

[8] Y.-G. Ha, J.-C. Sohn, Y.-J. Cho and H. Yoon "Towards ubiquitous robotic companion: design and implementation of ubiquitous robotic service framework," Journal of Advanced Computational Intelligence and Intelligent Informatics, vol. 27, no. 6, pp. 666-676, 2005, doi: 10.4218/etrij.05.0105.0138.

[9] B. Kehoe, S. Patil, P. Abbeel and K. Goldberg, "A Survey of Research on Cloud Robotics and Automation," in IEEE Transactions on Automation Science and Engineering, vol. 12, no. 2, pp. 398-409, April 2015, doi: 10.1109/TASE.2014.2376492.

[10] G. Hu, W. P. Tay and Y. Wen, "Cloud robotics: architecture, challenges and applications," in IEEE Network, vol. 26, no. 3, pp. 21-28, May-June 2012, doi: 10.1109/MNET.2012.6201212.

[11] B. Qureshi and A. Koubâa, "Five traits of performance enhancement using cloud robotics: a survey," Procedia Computer Science, vol. 37, pp. 220-227, 2014, doi: 10.1016/j.procs.2014.08.033.

[12] K. Kamei, S. Nishio, N. Hagita and M. Sato, "Cloud networked robotics," in IEEE Network, vol. 26, no. 3, pp. 2834, May-June 2012, doi: 10.1109/MNET.2012.6201213.

[13] G. Mohanarajah, D. Hunziker, R. D'Andrea and M. Waibel, "Rapyuta: A Cloud Robotics Platform," in IEEE Transactions on Automation Science and Engineering, vol. 12, no. 2, pp. 481-493, April 2015, doi: 10.1109/TASE.2014.2329556.

[14] J. Salmerón-Garc1'a, P. Íñigo-Blasco, F. D1'az-del-R1'o and D. Cagigas-Muñiz, "A Tradeoff Analysis of a CloudBased Robot Navigation Assistant Using Stereo Image Processing," in IEEE Transactions on Automation Science and Engineering, vol. 12, no. 2, pp. 444-454, April 2015, doi: 10.1109/TASE.2015.2403593.

[15] A. R. Chowdhury, "IoT and robotics: a synergy," PeerJ Preprints; 5: e2760v1, 2017, doi: 10.7287/peerj.preprints.2760v1.

[16] P. Simoens et al., "Internet of Robotic Things: Context-Aware and Personalized Interventions of Assistive Social Robots (Short Paper)," 2016 5th IEEE International Conference on Cloud Networking (Cloudnet), 2016, pp. 204207, doi: 10.1109/CloudNet.2016.27.

[17] H. G. C. Ferreira, E. Dias Canedo and R. T. de Sousa, "IoT architecture to enable intercommunication through REST API and UPnP using IP, ZigBee and arduino," 2013 IEEE 9th International Conference on Wireless and Mobile Computing, Networking and Communications (WiMob), 2013, pp. 53-60, doi: 10.1109/WiMOB.2013.6673340.

[18] D. Miorandi, S. Sicari, F. De Pellegrini and I. Chlamtac,"Internet of things: Vision, applications, and research challenges," Ad Hoc Networks, vol. 10, no. 7, pp. 1497-1516, 2012, doi: 10.1016/j.adhoc.2012.02.016.

[19] R. S. Batth, A. Nayyar and A. Nagpal, "Internet of Robotic Things: Driving Intelligent Robotics of Future Concept, Architecture, Applications and Technologies," 2018 4th International Conference on Computing Sciences (ICCS), 2018, pp. 151-160, doi: 10.1109/ICCS.2018.00033.

[20] N. K. Agrawal, V. K. Singh, V. S. Parmar, V. K. Sharma, D. Singh and M. Agrawal, "Design and Development of IoT based Robotic Arm by using Arduino," 2020 Fourth International Conference on Computing Methodologies and Communication (ICCMC), 2020, pp. 776-780, doi: 10.1109/ICCMC48092.2020.ICCMC-000144.

[21] L. Romeo et al., "Automated Deployment of IoT Networks in Outdoor Scenarios using an Unmanned Ground Vehicle," 2020 IEEE International Conference on Industrial Technology (ICIT), 2020, pp. 369-374, doi: 10.1109/ICIT45562.2020.9067099.

[22] A. Jangid, P. K. Dubey and B. R. Chandavarkar, "Security issues and challenges in Healthcare Automated Devices," 2020 International Conference on COMmunication Systems \& NETworkS (COMSNETS), 2020, pp. 1923, doi: 10.1109/COMSNETS48256.2020.9027291.

[23] S. Namani and B. Gonen, "Smart Agriculture Based on IoT and Cloud Computing," 2020 3rd International Conference on Information and Computer Technologies (ICICT), 2020, pp. 553-556, doi: 10.1109/ICICT50521.2020.00094.

[24] C. Chung and J. P. Jeong, "A Design of IoT Device Configuration Translator for Intent-Based IoT-Cloud Services," 2020 22nd International Conference on Advanced Communication Technology (ICACT), 2020, pp. 52-56, doi: 10.23919/ICACT48636.2020.9061282.

[25] F. Fellir, A. El Attar, K. Nafil and L. Chung, "A multi-Agent based model for task scheduling in cloud-fog computing platform," 2020 IEEE International Conference on Informatics, IoT, and Enabling Technologies (ICIoT), 2020, pp. 377-382, doi: 10.1109/ICIoT48696.2020.9089625.

[26] N. K. Agrawal, V. K. Singh, V. S. Parmar, V. K. Sharma, D. Singh and M. Agrawal, "Design and Development of IoT based Robotic Arm by using Arduino," 2020 Fourth International Conference on Computing Methodologies and Communication (ICCMC), 2020, pp. 776-780, doi: 10.1109/ICCMC48092.2020.ICCMC-000144.

[27] S. G.V.K., S. V. D., A. T., A. P., J. Bapat and D. Das, "Robotic Extension to IoT Testbed for Indoor Environment Supervision," 2019 Third International conference on I-SMAC (IoT in Social, Mobile, Analytics and Cloud) (ISMAC), 2019, pp. 29-35, doi: 10.1109/I-SMAC47947.2019.9032498. 